ÉGYPTE

monde arabe

\section{Égypte/Monde arabe}

4-5| 2001

L'Égypte dans le siècle, 1901-2000

\title{
D'un journalisme l'autre : contours d'une identité professionnelle et évolution de son rapport au pouvoir politique 1923-1970
}

\section{Sonia Temimi}

\section{CpenEdition}

Journals

Édition électronique

URL : https://journals.openedition.org/ema/864

DOI : $10.4000 /$ ema.864

ISSN : 2090-7273

Éditeur

CEDEJ - Centre d'études et de documentation économiques juridiques et sociales

Édition imprimée

Date de publication : 30 juin 2001

Pagination : 81-101

ISBN : 2-87027-963-9

ISSN : 1110-5097

Référence électronique

Sonia Temimi, «D'un journalisme l'autre : contours d'une identité professionnelle et évolution de son rapport au pouvoir politique 1923-1970», Égypte/Monde arabe [En ligne], 4-5 | 2001, mis en ligne le 08 juillet 2008, consulté le 07 juillet 2022. URL : http://journals.openedition.org/ema/864 ; DOI : https:// doi.org/10.4000/ema.864

Ce document a été généré automatiquement le 7 juillet 2022.

Tous droits réservés 


\title{
D'un journalisme l'autre : contours d'une identité professionnelle et évolution de son rapport au pouvoir politique 1923-1970
}

\author{
Sonia Temimi
}

1 Cet article a pour objet l'étude des changements qui ont affecté l'identité professionnelle des journalistes en Égypte et leur rapport au pouvoir politique entre la période dite «libérale» et la période nassérienne. Dans cette optique, deux personnages emblématiques ont été choisis : Muhammad Husayn Haykal (1888-1956) et Muhammad Hasanayn Haykal (1923). Exceptionnels à quelques titres, ils n'en demeurent pas moins représentatifs d'un «être journalistique " à deux périodes différentes. Le cadre temporel délimitant ce travail se situe entre 1923, date de la proclamation de la Constitution et de la naissance de la presse partisane, et 1970, celle de la mort de Nâsir [Nasser] et, avec lui, d'une certaine idée du pouvoir et de la place que doivent y occuper les journalistes.

2 L'étude de la presse égyptienne entre la période dite libérale et la période nassérienne révèle en effet l'évolution du statut de ceux qui l'écrivent. Mais il sera moins question ici de comparer les deux personnages que d'essayer de rendre compte des diverses significations que recoupe le terme «journaliste" d'une époque à l'autre. Comment chacun des deux personnages a-t-il incarné l'idée de la profession?

3 Il s'agira également d'analyser l'évolution du rapport de ces deux personnages au politique car si l'un appartient à un parti et en défend les idées, l'autre n'a pas d'affiliation politique claire mais suit un homme et contribue à développer son idéologie et à la mettre en forme. La Révolution de 1952 semble avoir constitué la grande rupture entre deux statuts du journalisme en Égypte. Avant 1952 et pendant la période "libérale ", c'était la figure du journalisme critique, partisan, en concurrence avec d'autres tendances constituant le paysage politique égyptien. Après 1952, c'est la naissance du journalisme d'État dans lequel le journaliste est enrôlé au service du 
pouvoir politique, avec pour tâche principale de justifier et de diffuser les idéologies et les politiques des dirigeants.

4 Les formations et carrières des deux personnages seront passées en revue en deux parties distinctes, jalonnées d'événements politiques susceptibles de rendre leurs parcours plus intelligibles. On notera que la partie consacrée à Hasanayn Haykal contient plus de détails, non qu'il s'agisse d'une préférence qui lui serait donnée, mais tout simplement parce qu'il se situe dans un double entre-deux, journalistique et politique

Mais avant d'aborder le sujet, quelques précisions s'imposent.

6 Les deux personnages choisis pour cette étude sont, certes, tous deux journalistes mais ils n'exercent pas leur métier de la même façon, c'est-à-dire qu'ils ne représentent pas le même type de journalisme. Si celui du premier se veut érudit et savant, le deuxième, en revanche, fait du journalisme professionnel, c'est un homme de terrain. Là, il serait tentant de coller à Husayn Haykal l'étiquette de «publiciste». Mais, les deux définitions de ce mot " écrivain politique dans le langage du XVIII et du XIXe siècle » et «au XIXe siècle, journaliste spécialisé dans l'information concernant l'actualité politique » (Balle, 1998, p. 198) peuvent s'appliquer indifféremment aux deux hommes. Il serait plus pertinent, toutes proportions gardées et nuances apportées, de les distinguer comme appartenant à deux écoles différentes, l'école française et l'école américaine de journalisme.

7 Par certains aspects de l'exercice de la profession, on peut avancer l'idée que Husayn Haykal fait du journalisme à la française, ce qui requiert une compétence littéraire, «faite de talent polémique, de pyrotechnie rhétorique » et où « la majorité des titres s'identifient à des sensibilités politiques, plus tard à des partis [...]. L'excellence professionnelle s'y fonde sur la maîtrise et le brio du style, la capacité à défendre une ligne éditoriale. Les contenus rédactionnels [...] traduisent le poids du commentaire, d'un méta-discours sur l'actualité qui privilégie l'expression des opinions, transforme l'événement en prétexte à exercices de style brillants et désinvoltes » (Neveu, 2001, p. 14). Parmi les publications auxquelles sont associées le nom de Husayn Haykal, deux étaient liées à son parti politique (ce qui sera expliqué plus loin dans la partie qui lui est consacrée). Il était chargé de défendre les idées du parti face aux attaques des autres journaux partisans, surtout ceux du Wafd. On lui doit également quelques polémiques restées célèbres, notamment avec Taha Husayn, pour la beauté du style avec lequel les deux hommes s'exprimaient et leur façon singulière de construire un argumentaire solide et truffé de références savantes. Ne perdons pas de vue que ce personnage a poursuivi des études en France et qu'il a été un lecteur assidu des auteurs français et un observateur impressionné par la richesse de la vie culturelle française.. Par ailleurs, les journalistes français au XIXe siècle «ne vivent pas leur activité comme un métier à part entière avec ses savoir-faire propres, sa logique de carrière. Travailler pour un journal est une position d'attente vers les vraies carrières de la littérature et de la politique " (Neveu, 2001, p. 12). Husayn Haykal commence par assumer la rédaction des journaux du parti, en devient le président plus tard et occupe plusieurs fois le poste de ministre. Mais il ne cesse de faire des allers-retours entre le monde de la presse et celui de la politique. C'est pour toutes ces raisons qu'il a semblé que Husayn pouvait être associé à l'école française dans sa façon de faire du journalisme.

8 Hasanayn Haykal, quant à lui, appartiendrait à l'école américaine où la dimension de collecte de l'information est importante. «Être journaliste suppose un rapport au 
terrain, la constitution d'un carnet d'adresses, des savoirs-faire liés à la prise de notes, au recoupement de l'information, à la maîtrise de la situation de l'entretien. [...] En associant ainsi à la pratique journalistique des tâches, des compétences, une écriture irréductible à celle d'activités préexistantes (écrivains, personnel politique), le modèle anglo-américain en fait une activité pensable comme une profession à part entière, ouvrant à ses praticiens des perspectives de carrière" (Neveu, 2001, p. 10). Ce personnage, qui n'a exercé que ce métier', a été formé par un journaliste américain et a travaillé pendant une dizaine d'années avec les frères Amîn, fondateurs de la première maison de presse égyptienne et très influencés par la tradition journalistique angloaméricaine. Son domaine de prédilection est le terrain. Il est l'un des premiers à avoir instauré la tradition de reporter dans le journalisme égyptien et à avoir parcouru le monde cinq années d'affilée pour couvrir les conflits les plus importants. Son carnet d'adresses est digne des grands journalistes (ce qui lui sera fort utile plus tard, lorsqu'il sera proche de Nâsir), et rares sont les chefs d'État arabes de l'époque qui n'aient été interviewés au moins une fois par lui. Changeant à plusieurs reprises de journal lorsqu'il sentait qu'il en avait gravi tous les échelons, il relève le défi de la reprise d'un journal en faillite, al-Ahrâm, et travaille pendant près de dix-huit ans à en faire le premier quotidien égyptien, voire arabe. C'est la dernière étape de son ascension professionnelle, à savoir l'entière responsabilité de la direction d'un journal. Certes sa relation avec Nâsir a beaucoup contribué à en faire, pendant un temps, le premier journaliste égyptien mais il n'en demeure pas moins un journaliste professionnel de talent. Toutefois, il faut noter qu'au moment de son éviction de la tête d'al-Ahrâm, il avait délaissé depuis un certain temps déjà son rôle d'« historien de l'instant " pour se transformer en porte-parole d'un régime. Actuellement, Hasanayn Haykal n'appartient officiellement à aucun journal mais il commente régulièrement l'actualité politique. Il reste un personnage important sur la scène intellectuelle égyptienne, une référence dont les commentaires sont attendus par le grand public et le succès commercial de ses ouvrages en témoigne. Ayant commencé sa carrière et bâtit son succès sur l'exercice de la profession de journaliste, ne voudrait-il pas terminer son parcours en devenant une sorte de «journaliste-penseur des temps modernes», un commentateur de l'actualité politique en valorisant la prise de distance?

Muhammad Husayn Haykal, presse partisane et élite économico-intellectuelle

9 Né en 1888, Muhammad Husayn est issu d'une grande famille de propriétaires terriens. Après une licence de droit, il part à Paris en 1909 pour y préparer une thèse sur La dette publique de l'Égypte. Il se dit très impressionné par la richesse de la vie culturelle et sociale françaises. Sa première collaboration à un journal remonte à l'époque qui a précédé son départ. Il écrit dans la revue du parti de l'Umma, al-Garîda qui appartient à son parent Lutfî al-Sayyid', son maître à penser.

10 Il continue d'y écrire lors de son séjour en France et en est le correspondant pendant ses vacances scolaires. En rentrant, il est déjà intégré dans le milieu politique et intellectuel de l'époque étant donné que le siège d'al-Garîda, sert de lieu de rencontre à des personnalités prestigieuses. Après l'arrêt de la revue, il contribue à la réussite d'alSufûr et publie des études dans différents autres journaux'. Durant la même période, il est avocat dans un bureau à al-Mansoura et enseigne à l'Université du Caire toutes les semaines

11 Bien que cette version soit actuellement contestée, il fut considéré pendant longtemps comme un romancier avant-gardiste de la littérature arabe moderne. Il fait paraître en 
1914 sous le pseudonyme de "Paysan égyptien" son roman Zaynab. Il y traite de la situation de la paysannerie égyptienne et son ouvrage a pour but de revaloriser cette catégorie à laquelle les membres de l'aristocratie turque gouvernante n'accordent pas le « respect qui lui est dû ». Il publie un livre en 1921 sur Jean-Jacques Rousseau. Entre 1935 et 1945, il écrit une série d'ouvrages sur les saints de l'Islam".

En raison des liens qui l'unissent à Lutfî al-Sayyid, Husayn Haykal est très proche du parti de l'Umma et de son idéologie. Bien que trop jeune à l'époque pour en être un membre actif, les idées du parti façonnent sa vision politique. C'est Lutfî al-Sayyid qui lui sert de maître en lui expliquant les " principes de la liberté tels que les ont compris les gens du XIX' siècle en Europe » (Husayn Haykal, 1990, Vol. I, p. 30) et qui l'incite à se tourner vers des lectures autres que la littérature arabe pour élargir ses horizons. En lui transmettant sa passion de la philosophie, des débats d'idées et de la politique, il lui transmit également la tendance à l'élitisme qui caractérise son mouvement. À ses camarades étudiants scoptiquer à l'idée avancée par le parti selon laquelle l'Égypte doit se libérer de l'influence ottomane, Haykal rétorque que "seule l'élite qui a reçu un enseignement supérieur et non le peuple " est en mesure de comprendre une telle revendication (Husayn Haykal, 1990, Vol I, p. 30). Muhammad Husayn Haykal, comme une grande partie des «hommes éclairés » composant la tendance libérale, considère que si l'Europe rationnelle a atteint le plus haut degré de civilisation, c'est parce qu'elle a rompu définitivement avec un ancien système social dominé par les institutions religieuses. Chez Husayn Haykal, cette conviction, qui va paradoxalement de pair avec une tendance au conservatisme social, le mène à penser que seuls les "grands hommes » (à comprendre au sens de héros)" sont capables de guider les peuples «noyés dans l'ignorance, la religiosité et les traditions». Partagé entre un sentiment de responsabilité envers le peuple « ignorant et non éduqué », il n'en demeure pas moins conscient d'appartenir à une élite dont il ne se prive pas de défendre les privilèges. Un moment défenseur de la généralisation de l'enseignement primaire" ${ }^{\text {, }}$ il se ravise et opte au contraire pour la limitation de l'enseignement supérieur afin de permettre l'autoreproduction de la classe des privilégiés.

Une autre thématique récurrente dans ses écrits est son rapport au peuple. Son horreur de l'emportement des foules" et de la polarisation des extrêmes le mènent, comme ses compagnons, à s'opposer au Wafd et à ce qu'il considère comme «un appel démagogique à la populace » (Laurens, 1995, p. 101).

La première affiliation à proprement parler de Husayn Haykal à un parti politique remonte à 1919 lorsqu'il décide, avec des amis rencontrés en France, de créer le Parti démocrate" (en réaction à la constitution du Wafd) mais son existance est brève. Bien que ses membres s'accordent à adopter des principes généraux comme la liberté, l'égalité, une justice neutre et le principe de l'auto détermination des peuples comme fondement de leur parti, trop de divergences idéologiques en rendent la gestion impossible.

15 C'est alors que le Parti libéral-constitutionnel est créé. Haykal joue un rôle actif dans la diffusion de ses idées. Ses adhérents, pour la plupart anciens ministres, hauts fonctionnaires, grands propriétaires terriens, s'efforcent d'introduire dans la vie politique égyptienne un élément de stabilité et de modération. Ennemis déclarés des égarements de la passion et également éloignés de tous les extrémismes, ils redoutent par dessus tout l'agitation de la rue. Ardents défenseurs de la Constitution, ils rêvent d'un régime monarchique constitutionnel fonctionnant sans heurts sur le modèle de 
celui de la Grande-Bretagne. Mais le parti sera l'allié de «l'aile moderne », industrielle et technocratique de la bourgeoisie qui, " au lieu d'être le porte-parole d'un parlementarisme plus efficace, d'une rationalisation de la société, d'une pensée plus soucieuse de progrès réel, d'une intransigeance politique qu'aurait pourtant justifié son rôle d'avant-garde de la société égyptienne en pleine mutation, va être l'instrument choisi de la réaction sur le plan politique » (Abdel-Malek, 1962, p. 23). Malgré leur attachement au régime constitutionnel, les membres du parti n'hésitent pas à en trahir les principes afin d'arriver au pouvoir". Husayn Haykal vit alors une "crise doctrinale » mais continue de supporter les libéraux-constitutionnels membres du gouvernement contre les attaques du Wafd.

16 En 1922, il devient leur porte-parole en assumant la rédaction du quotidien al-Siyâsa. Au début de sa création, une expérience nouvelle est tentée, celle de ne recruter les rédacteurs et délégués que parmi les diplômés universitaires. Ne réussissant pas vraiment à former une équipe de délégués à la hauteur de ses attentes, ses rédacteurs constituent néanmoins une petite élite (ils deviennent ministres, ambassadeurs, hommes politiques ou hauts fonctionnaires). Outre son rôle de porte-parole des libéraux-constitutionnels (surtout dans le quotidien), Husayn Haykal contribue très fortement au succès de l'hebdomadaire de ce même parti al-Siyâsa al-usbûciyya à partir de 1926". Revue littéraire, elle regroupe une pléiade d'écrivains de renom."

17 À côté de cela, il entame une carrière politique. Il entre dans six gouvernements entre 1937 et $1945^{\prime \prime}$. Il applique un programme de déconcentration au ministère de l'Instruction publique. Notons qu'il est à l'origine du contrôle du ministère sur les écoles étrangères. Il y impose l'enseignement de l'arabe, de l'histoire et de la géographie de l'Égypte. Il veille également à la création de bibliothèques dans tous les lycées et dans certaines écoles primaires afin d'encourager les jeunes générations à la lecture.

18 Afin de justifier l'action du gouvernement dont il fait partie et qui ne tient pas toujours ses promesses électorales, Husayn Haykal avance que la vision des problèmes que peut avoir un ministre différe de celle d'un journaliste. Elle est plus réaliste mais seuls ceux qui détiennent le pouvoir sont en mesure d'en apprécier les difficultés (Husayn Haykal, 1990, vol. II, p. 10-11).

19 Il est élu président de son parti en 1943. Il sera également président du Sénat entre le 19 janvier 1945 et le 17 juin 1950. Pendant cette période, il rencontre le Roi (qui le consulte sur quelques questions relatives à la vie politique égyptienne). Mais sa fonction étant dépendante de la bonne entente qu'il a avec ce dernier et de son bon vouloir, il en est démis sans justification.

Husayn Haykal fait partie de l'élite intellectuelle qui, jusque dans les années trente, porte le flambeau de la modernisation de l'Égypte à travers l'introduction des concepts européens. Cette dimension est d'ailleurs reprise par les partis politiques minoritaires ou les indépendants qui se partagent le pouvoir ${ }^{10}$. De par leur caractéristique minoritaire, ils ne peuvent compter sur le soutien populaire qui est tout entier acquis au Wafd. Il en résulte que même la tendance libérale trahit ses idéaux en falsifiant quelques fois les élections afin d'obtenir une majorité parlementaire ou de s'indigner de la supercherie lorsque les résultats donnent le Wafd gagnant. La seule solution pour parvenir au pouvoir et en évincer le Wafd est de s'allier tantôt avec le Palais et tantôt avec les Anglais. Beaucoup de prérogatives sont également laissées au Roi qui intervient directement dans les décisions du gouvernement. Les libéraux- 
constitutionnels s'en indignent très peu, sachant tous les avantages qu'ils peuvent tirer d'une telle alliance. En vingt-neuf ans de « libéralisme », le Wafd, ce parti majoritaire,

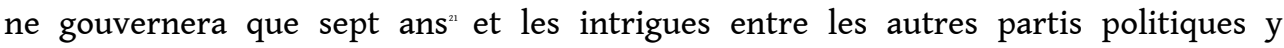
contribuent grandement. Entre 1923 et 1952, aucune législature n'est menée à terme. Bien que tous les "accessoires" du système libéral soient réunis (Constitution, Parlement, partis politiques), les mécanismes en sont faussés à cause de la concurrence des partis minoritaires entre eux et avec le Wafd ainsi qu'à cause de la confrontation entre le Palais et le Wafd avec les Anglais en toile de fond. La presse partisane durant toute cette période se nourrit de cette concurrence et reflète un débat continuel portant sur les moyens que chaque parti veut mettre en œuvre pour parvenir à l'indépendance de l'Égypte.

S'agissant de la presse, Ibrâhîm 'Abdû recense la parution de 1146 publications (quotidiens, hebdomadaires, bi-mensuels, mensuels, semestriels...) entre 1798 et 1951 en Égypte ('Abdû, 1982, p. 335-342). Le chiffre est important mais il est à relativiser car beaucoup ont une durée de vie très limitée ou n'ont aucune influence. Ce chiffre comprend également la presse spécialisée, celle des provinces et celle des communautés étrangères installées en Égypte mais elles n'ont pas le même poids.

En ce qui concerne la presse partisane, plusieurs journaux soutiennent le Wafd et la politique de Sacd Zaghlûl. 'Abd al-Qâdir Hamza assume la rédation d'Al-Balâgh, " quotidien politique, littéraire et commercial », créé le 28 janvier 1923. Kawkab al-Sharq voit le jour le 21 septembre 1924 et son propriétaire, Ahmad Hâfid 'Awad', annonce qu'il travaillera avec acharnement pour réaliser l'indépendance totale de l'Égypte et du Soudan et le renforcement de la dimension orientale et islamique de l'identité égyptienne (Abdû, p. 214). Les saadistes sont défendus par le journal al-Asâs. Les partis du roi font paraître des journaux qui portent leurs noms : al-Ittihâd et al-Shacb qui n'ont pas beaucoup de poids sur la scène bien qu'ils bénéficient de la collaboration de journalistes connus (notamment 'Abd al-Qâdir al-Mâznî qui écrit dans al-Ittihâd). À côté de cette presse, il existe aussi des journaux indépendants : le quotidien al-Ahrâm, porteparole des gouvernements successifs de quelque bord qu'ils soient; al-Muqqattam, proanglais; l'hebdomadaire Rûz al-Yûsuf, al-Hilâl; les revues littéraires, al-Muqtataf, alÂdâb...

Husayn Haykal défend les libéraux-constitutionnels dans le quotidien al-Siyâsa puis dans l'hebdomadaire al-Siyâsa al-usbûciyya qui contribue énormément à dynamiser le mouvement de pensée égyptien du début du siècle. Les sujets les plus régulièrement abordés par al-Siyâsa al-usbûciyya sont ceux consacrés à la littérature ou au traitement des questions qui en découlent (critique littéraire, traduction de nouvelles en majorité françaises, nouvelles égyptiennes, pièces de théâtre, études littéraires). Viennent ensuite les articles de politique intérieure puis à ceux consacrés aux sciences humaines et expérimentales, en plus de quelques sujets ponctuels dictés par l'actualité du moment. Husayn Haykal y écrit 325 articles dont 227 consacrés à des sujets littéraires et 108 portant sur la politique (Muhammad, 1996, p. 218)". Une certaine objectivité caractérise al-Siyâsa al-usbû́iyya. Ses auteurs contribuent à moderniser la langue arabe en optant pour un vocabulaire standard.

Sayyid Muhammad divise l'histoire de la revue en quatre périodes. De mars à octobre 1926, deux principaux sujets sont traités : la littérature et la définition des hommes de lettres avec des articles notamment de Taha Hussein. Haykal aborde la question des 
rapports entre la religion et la science et il avance l'idée qu'il n'y a pas de contradictions ou concurrence entre les deux mais que la concurrence se situe plus au niveau des hommes de religion et ceux des sciences pour le pouvoir, ce que réfutent Mahmûd 'Azmî et de Sayyid cAfîfî. La deuxième période, 1926-1931, est la plus importante. Y sont perceptibles des changements dans la pagination et les rubriques: réduction du nombre de pages, collaboration de nouveaux écrivains (May pour la rubrique féminine et sociale, Fikrî Abâza). C'est durant cette période que les rubriques prennent leur forme définitive. Des correspondants à l'étranger élargissent la palette des sujets abordés (Lettre de Palestine, de Turquie, d'Inde, de Londres, de Paris...). Les questions moyen-orientales prennent de plus en plus d'importance. Des numéros sont consacrés à des personnalités de l'histoire égyptienne contemporaine. Enfin, Haykal publie des extraits de ses ouvrages. L'intérêt de la revue se porte sur des sujets divers : l'éducation (il faut la généraliser mais aucune planification n'a été faite), le besoin d'une encyclopédie générale arabe et de nouveaux dictionnaires ; la littérature pré-islamique, la poésie et la prose. S'ensuit un débat sur la littérature nationale. À ce propos, sont préconisés l'arrêt des traductions et la création d'une littérature égyptienne moderne capable d'exprimer les différents aspects de la vie quotidienne et plus généralement de la société. Pour cela, quelle langue faut-il utiliser, l'arabe littéraire ou le dialecte? Sacd Mâlikî aborde la liberté d'expression en Égypte et cite l'exemple de 'Alî ‘Abd al-Râziq" et Taha Husayn" pour leurs ouvrages respectifs avec en arrière-plan l'exemple européen et la renaissance. La réforme d'al-Azhar, l'Alliance orientale font également partie des centres d'intérêts de la revue. À partir du 30 juin 1928, l'influence de l'appartenance politique du journal, discrète jusque-là, commence à se faire sentir. Sidqî arrête le quotidien al-Siyâsa, l'hebdomadaire devient alors l'unique moyen d'expression des libérauxconstitutionnels.

Arrêtée pendant six ans, la revue paraît à nouveau à partir de janvier 1937 et entame sa troisième période qui s'étend jusqu'en janvier 1942. Les sujets varient entre religion, poésie, philosophie, littérature égyptienne et arabe, politique arabe, quelques sujets sur des problèmes sociaux, la science, le droit, l'agriculture. Fin décembre 1937, Haykal quitte le journal suite à sa nomination comme ministre. À son retour, il prend la responsabilité de modifier des rubriques et de re-dynamiser la revue. Les personnes qui y ont collaboré dans le passé y contribuent à nouveau. Mais la caractéristique principale de la revue à cette époque est le passage à un discours partisan plus affirmé et la relégation des préoccupations littéraires au second plan. Pendant la Seconde Guerre mondiale, beaucoup de sujets traitent de cette dernière sous un angle proanglais mais avec les intérêts de l'Égypte pour toile de fond. $Y$ est noté également un intérêt pour les journalistes et leur syndicat après sa création en 1941 (compte rendu des réunions et des discussions). L'Académie de la langue arabe suscite un vif intérêt car elle remet à l'ordre du jour le débat sur les dialectes.

28 C'est durant la quatrième période, qui va de janvier 1942 à mai 1949, que l'appartenance politique de la revue est la plus flagrante. On y lit beaucoup d'attaques contre le Wafd et al-Nahhâs en plus de nouvelles se rapportant au parti libéralconstitutionnel stricto sensu. Le quotidien al-Siyâsa réapparaît en décembre 1944. Husayn Haykal s'y consacre en délaissant l'hebdomadaire de plus en plus pauvre en débat. Il continue de traiter de questions littéraires. On voit aussi apparaître des articles d'informations événementielles. Sayyid Muhammad estime que cela est 
révélateur de la volonté d'étoffer une revue en manque de sujets et d'auteurs de renom (Muhammad, 1996, p. 202). Mais peut-être doit-on y voir également un effet de l'évolution de la presse et de sa professionnalisation. La concurrence des autres journaux est plus forte. À la fin de cette période, beaucoup d'articles portent sur la guerre de Palestine.

L'histoire de la revue a été jalonnée par des numéros spéciaux consacrés à des personnalités politiques et littéraires avec, dans la quatrième période, un accent mis plus spécifiquement sur des membres du parti. Husayn Haykal affirme à plusieurs reprises son respect de la diversité des opinions. Preuve en est que la revue s'attache à publier des avis opposés et qu'il soutient énergiquement ‘Ali ‘Abdel Râziq lors de la parution de son ouvrage.

Cette presse partisane a animé de riches débats idéologiques. Pour ne prendre qu'un seul exemple, on dira que, si Taha Husayn écrit sur les avancées réalisées lors des guerres et leurs bienfaits pour l'humanité, c'est justement pour que Husayn Haykal prenne la position contraire et démontre leurs effets néfastes en reprenant point par point l'argumentaire de Taha Husayn ${ }^{*}$. Mais, on a pu quelquefois avoir le sentiment qu'elle était le reflet d'une petite élite politique partageant les mêmes intérêts. La frontière entre leurs différences est parfois difficile à établir. En créant son parti AlShacb, Ismâ̂îl Sidqî propose à Husayn Haykal d'assumer la rédaction de son journal contre une forte rémunération (Muhammad, 1996, p. 59). Les libéraux-constitutionnels se lient avec le Wafd en 1926 afin de renforcer l'opposition à leur allié de la veille, Ahmad Zîwar du parti d'al-Ittihâd. Moins de dix ans après l'instauration du régime libéral, se pose la question de la crédibilité qu'avaient tous ces partis pour le peuple. Un jour alliés, ennemis le lendemain, leur presse partisane illustre ces changements de positionnement. Mais qui touche-t-elle vraiment? Combien de personnes issues des couches populaires s'intéressent à ces débats surtout si l'on tient compte du fait que jusque dans les années 1920, 25 à $30 \%$ de la population masculine étaient scolarisés ? Seule une minorité s'est enrichie, la classe moyenne (représentée par les petits fonctionnaires, les professions libérales), celle qui a pu intégrer l'université à partir du milieu des années 1935.

31 Parallèlement sur la scène politique, les gouvernements se succèdent mais sans avancée notable dans les négociations avec les Anglais. La Seconde Guerre mondiale marque une déception pour le nationalisme égyptien, l'espoir de l'évacuation des troupes anglaises se réduit. La vie politique est le théâtre d'une montée de la violence. Se fait alors un réaménagement de la scène politique avec l'apparition de nouvelles formations, accompagnées d'une presse critique, plus professionnelle issue de maisons de presse égyptiennes nouvellement créées.

Muhammad Hasanayn Haykal : entre « âge d'or » de la presse et étatisation du métier Muhammad Hasanayn est né dans le vieux quartier populaire cairote d'al-Husayn en 1923, d'une famille de petits commerçants. Cette appartenance sociale est quelquefois utilisée par ses détracteurs pour diagnostiquer un complexe d'infériorité chez Haykal".

Il obtient un diplôme dans une école de commerce en Égypte en 1939. Réalisant que son diplôme ne suffisait pas pour un début même modeste, il semblerait qu'il se soit inscrit dans une école française en Égypte pour poursuivre des études en économie, en plus d'un diplôme en droit par correspondance". Il prend également des cours à l'Université américaine". En même temps qu'il entame une carrière dans le journalisme, la scène 
politique égyptienne est le témoin d'une recrudescence de la violence politique s'accompagnant d'un durcissement de ton dans la presse.

Le 4 février 1942, Mustafâ al-Nahhâs est ramené au pouvoir "sur la pointe des baïonnettes anglaises $»^{32}$ comme dira l'opposition. En paraissant légitimer cette intrusion britannique dans la souveraineté égyptienne, la direction du Wafd se discrédite fortement aux yeux de l'opinion. Quelques mois plus tard, le 26 mai 1942, Makram 'Ubayd est exclu du cabinet au sein duquel il détient le portefeuille des Finances. Il crée son parti appelé Bloc wafdiste indépendant et son journal al-Kutla. Cette nouvelle scission, en raison même de la personnalité de son instigateur, ébranle fortement les assises du Wafd dont Makram 'Ubayd était le secrétaire général depuis des années. Connaissant tous les rouages du parti et jouissant d'une solide réputation d'intégrité, ses attaques contre le Wafd n'en sont que plus fortes. En 1943, il rédige un pamphlet qui circule clandestinement sous le titre de "Livre noir » dans le but de jeter le doute sur la probité d'al-Nahhâs et sur l'intégrité de son entourage. Il atteint son objectif et l'opinion publique accorde plus de crédit aux attaques de l'opposition contre le gouvernement wafdiste.

À la fin de la Seconde Guerre mondiale, la question sociale devient un leitmotive des gouvernements successifs. Ce changement s'opère sous l'influence des groupements de gauche, de plus en plus nombreux. On assiste également à l'émergence d'autres formations politiques se disant capables de proposer une alternative. Ahmad Husayn, dirigeants des chemises vertes du parti Jeune Égypte, - le futur Parti national islamique (1940), puis Parti socialiste (1946) -, mène campagne contre les grands féodaux. Les Frères musulmans de Hasan al-Bannâ organisent une série d'attentats meurtriers entre 1945 et $1948^{\prime \prime}$, ce qui pousse le gouvernement saadiste d'al-Nuqrâshî à dissoudre l'organisation le 8 décembre 1948. Vingt jours plus tard, al-Bannâ est assassiné. Né d'une alliance entre intellectuels wafdistes, nationalistes, communistes et syndicats ouvriers, le Comité national des ouvriers et étudiants voit le jour en 1946. Ses méthodes sont celles de l'action de masse: comités nationaux d'entreprises, de rues, de professions, manifestations, grèves... La police ouvre le feu sur les manifestants à plusieurs reprises". L'agitation de la rue est plus importante (49 grèves en 1950). À partir de 1951 et ayant perdu tout espoir d'une évacuation britannique négociées, une guérilla s'organise dans la zone du canal de Suez.

Cette montée de la violence politique s'accompagne d'un durcissement de ton dans la presse. Les critiques sont plus directes et virulentes. Les années qui s'étendent de 1940 à la Révolution de 1952 sont considérées comme «l'âge d'or » de la presse critique égyptienne. En 1940, plus de cent quotidiens et hebdomadaires, majoritairement arabophones, paraissent au Caire. De nouvelles maisons d'éditions et sociétés de presse égyptiennes voient le jour, faisant concurrence aux éditeurs syro-libanais et insufflant un renouveau dans les techniques journalistiques : la mise en page, le choix des articles, le style d'écriture. En 1937, le Conseil des ministres discute de la création d'un département de journalisme à l'université. Le syndicat des journalistes est créé en 1941. Deux nouvelles presses, l'une partisane et l'autre indépendante, sont nées. De nouveaux journaux wafdistes voient le jour dont les deux plus importants sont le quotidien sawt al-Umma dirigé par Muhammad Mandûr (1946) et le premier quotidien égyptien, porteparole officiel du Wafd, al-Misrî (influencé par la presse britannique) et représentant la tendance de gauche au sein du Wafd et dirigé par 'Azîz Fahmî, Mahmûd et Ahmad Abul-Fath. Une importante presse communiste naît : al-Fagr al-Gadîd, Om Dourman, al-Talî‘a 
(1945 -1946) ; puis, al-Gamâhîr (1948), al-Malâyîn (1951). Il y a aussi la revue socialiste alIshtirâkiyya de Ahmad Husayn, les journaux al-Dacwâ et al-Ikhwân al-muslimûn des Frères musulmans. En 1944, Akhbâr al-Yawm (influencé par la presse américaine) vient renforcer les rangs de la presse indépendante.

Quelques faits sont largement débattus dans l'ensemble de la presse : les événements du 4 février 1942, le "Livre noir », la guerre en Palestine, le projet de lois de Istafân Bâsîlî visant à protéger le Roi contre toute critique et l'abrogation du traité de 1936 . Trois journaux surtout font figure de chef de file de la presse critique : Akhbâr al-Yawm, al-Misrî et Rûz al-Yûsuf. Les journaux de gauche jouent un rôle dans la critique de la situation économique, la défense des droits des travailleurs et des paysans. C'est l'époque où est lancé le slogan «La terre à ceux qui la travaillent » et où sont faites des propositions de redistribution des terres aux paysans s et l'imposition de nouvelles taxes aux plus riches pour une plus grande justice sociale.

Muhammad Hasanayn commence sa carrière en février 1942 à The Egyptian Gazette et part à El-Alamein apprendre le métier ${ }^{*}$. Après un très court passage à Rûz al-Yûsuf, il va travailler à Âkhir Sấc $a^{\text {n }}$ avec Muhammad al-Tâbi $\hat{1}^{s}$, qui est le premier à lui faire prendre conscience du rôle qu'il peut et doit jouer dans le milieu du journalisme arabophone. C'est à cette époque également qu'il réalise l'importance des dimensions égyptienne et arabe de son identité. Il suit les séances du Parlement, ce qui lui permet de comprendre le fonctionnement de la vie politique égyptienne et d'être au contact de ses acteurs les plus importants. Sur les conseils d'al-Tâbicî, il devient un spectateur assidu des pièces de théâtre, « espace important où s'expriment les aspirations du peuple à la liberté et à l'indépendance ».

Mais la véritable innovation de Haykal est d'avoir été correspondant de Akhbâr al-Yawm des frères Amîn" à l'extérieur de l'Égypte durant une longue période et d'avoir rompu avec la traditionnelle focalisation des rubriques internationales sur la Grande-Bretagne (pour les affaires politiques) et la France (pour les affaires culturelles). Il se démarque de ses confrères en réussissant à attirer l'attention de l'opinion publique sur d'autres parties du monde ${ }^{\prime \prime}$. C'est grâce à cette expérience qu'il se fait un nom sur la scène journalistique et intellectuelle égyptienne et il remporte trois fois d'affilée le prix de la presse «Farouk Premier », très prisé parmi les jeunes journalistes. Il réussit également à se faire connaître dans le milieu des hommes politiques par ses reportages sur la guerre de Palestine en 1948 intitulés « Le feu sur la terre sainte ». Parallèlement, il est en contact avec toute une génération de journalistes du monde entier et réussit à tisser un réseau considérable de connaissances, élément qui jouera largement en sa faveur.

Hasanayn Haykal fait partie de la génération qui assiste à l'essoufflement du régime parlementaire, à la multiplication des scandales qui touchent les différents partis et leurs dirigeants, une génération de déçus qui aspirent à un changement. Il est pourtant en contact avec les hommes politiques de la période libérale" et c'est peut-être cette proximité qui lui permet de comprendre les dysfonctionnements du système. Il est très marqué par la guerre de 1948 et par la réaction irrationnelle des pays arabes qui ne prennent pas leur adversaire au sérieux. Dans ses articles « Le feu sur la terre sainte " il analyse les raisons de la supériorité de l'ennemi. Al-Hilâlî, alors chef du gouvernement, le convoque pour lui dire à quel point ses écrits nuisent au moral de la nation (Shalabî, 1999, p. 33). Tous ces facteurs peut-être poussent Hasanayn Haykal à soutenir le mouvement révolutionnaire du 23 juillet 1952. 
41 Au début de cette nouvelle ère, la Révolution, qui incarne l'espoir d'un changement, est saluée par la presse. Une majorité de journalistes s'y rallie. Nâsir, encore dans l'ombre du général Néguib, est en contact avec des journalistes, notamment Ahmad Abu-l-Fath et Ihsân cAbd al-Quddûs qui prennent la défense du mouvement révolutionnaire. Pourtant, un incident illustre le type de relations qui va s'instaurer entre la presse et le pouvoir politique : les frères Amîn sont arrêtés, accusés d'entretenir des contacts douteux avec l'étranger. Quelques semaines après la révolution, Hasanayn Haykal s'attaque à la presse et appelle à son "auto-purification». Le syndicat le traduit en conseil disciplinaire. À cette époque déjà, il fait quatre propositions pour améliorer la situation de la presse, et notamment son organisation ${ }^{*}$. Au moment de la crise de mars 1954, deux tendances s'affrontent dans les colonnes des journaux. Soutenant les républicains libéraux, représentés par le général Néguib et Khâlid Muhyî al-Dîn, la première tendance est menée par al-Misrî, al-Qâhira, Rûz al-Yûsuf et al-Gumhûr al-Misrî. Un autre groupe, formé par al-Gumhûriyya et al-Akhbâr", soutient le colonel Nâsir qui finit par l'emporter. Le premier groupe paye alors le prix de sa prise de position. Le 4 mai 1954, al-Misrî, qui fait figure de porte-parole de l'ensemble des courants démocratiques, est interdit ; Ihsân 'Abd al-Quddûs est rappelé à l'ordre par quelques jours de prison après la publication de son article "La société secrète qui gouverne l'Égypte». En avril, une liste de 24 journalistes ayant reçu des financements occultes sous l'ancien régime est publiée. Le ton est donné et les bases de la relation entre le pouvoir et la presse sont établies.

La presse lance des slogans qui rencontrent un écho favorable bien au-delà des frontières égyptiennes : nation arabe, nationalisme arabe, union des Arabes, unité des peuples frères... slogans qui atteignent leur paroxysme au moment de l'unité syroégyptienne (1958-1961). Le journal al-Shacb (1956) est destiné à affirmer l'orientation pan-arabe de l'Égypte. Al-Masâ' (1956) est dirigé par Khâlid Muhyî al-Dîn, de retour d'exil, qui s'entoure d'une pléiade de jeunes intellectuels. Le journal devient la tribune des socialistes et progressistes égyptiens. Pour la deuxième fois, Nâsir et Khâlid Muhyî al-Dîn s'affrontent, et ce dernier finit par quitter le journal.

Sur le plan intérieur, le bien-être social et la juste répartition des richesses sont également devenus des leitmotive servant à justifier la privation des libertés politiques. Augurée par la réforme agraire et l'industrialisation de l'Égypte, cette quête de bienêtre donne au régime une orientation socialiste. Fleurissent alors des expressions telles " pour le peuple », «le bien du peuple », «l'intérêt du peuple », « au nom du peuple » qui, bien que servant à justifier des mesures répressives, valent à Nâsir un soutien populaire sans bornes. Par contre, aux intellectuels moins dupes, est avancée l'idée que l'édification d'une nouvelle société plus équitable requiert le choix difficile du sacrifice momentané des libertés politiques. Leur rôle consiste à jouer les intermédiaires entre le pouvoir et la société afin d'expliquer et de transmettre le projet sociétal du nouveau régime. Cette tâche revient en partie à Haykal. Mais essayons d'abord d'expliquer pourquoi Nâsir choisit Haykal qui, en 1952, est loin d'être le journaliste le plus proche de lui.

S'il n'est pas le journaliste le plus connu, Haykal est par contre l'une des seules figures non marquées politiquement au milieu de cette presse "corrompue " pour reprendre l'expression de l'époque. Bien qu'il travaille pour des journaux dont les prises de position en faveur de telle ou telle partie sont radicalement opposées (Akhir Sấa était wafdiste et Akhbâr al-Yawm très proche du Palais), cela ne l'empêche nullement 
d'afficher son opposition lorsqu'il le juge nécessaire". Mais il devient surtout l'un des seuls journalistes capables d'analyser la politique extérieure à une époque où celle-ci revêt de plus en plus d'importance pour l'Égypte.

Selon la version qu'il donne de ses rencontres et de ses relations avec Nâsir, il aurait été capable de l'influencer pour ce qui est de l'exécution de ses projets de coup d'État. Son analyse postulait que les Anglais n'auraient pas les moyens d'intervenir sur le terrain si le roi demandait leur aide". Le soir de la Révolution, il se trouve au siège du Commandement de la révolution et sert d'intermédiaire entre le chef du gouvernement Nagîb al-Hilâlî et le comité directeur.

Commence alors une relation politico-affective entre les deux hommes. Si Hasanayn Haykal voit en Nâsir un homme susceptible de mener l'Égypte vers la centralité qu'elle " mérite » sur la scène arabe et internationale, Nâsir trouve en lui la personne capable de transcrire avec élégance ses rêves et ses projets de grandeur. C'est ainsi que de leur première collaboration naît $L a$ philosophie de la révolution. Une fois passée la criseexamen de 1954, Nâsir s'assure définitivement de la fidélité de Haykal.

Hasanayn Haykal est avant tout une source d'informations pour Nâsir. Aux journalistes qui s'indignent pendant la conférence de Bandung de la rétention d'informations dont ils se sentent victimes, Nâsir rétorque : «il est ma source d'informations " et non le contraire (Hammûda, 2000, p. 180). En raison des liens qui l'unissent aux journalistes, éditeurs et hommes politiques dans le monde, il obtient plusieurs fois des informations concernant l'Égypte et son leader que les ambassades égyptiennes n'ont pas.

Sur le plan professionnel, 1957 marque le point de départ du «mythe » de Hasanayn Haykal puisqu'il finit par accepter l'offre des propriétaires du journal al-Ahrâm" et en devient le rédacteur en chef. Entre le 1er août 1957 et le 2 février 1974, le journal se transforme, se modernise. Au moment de sa prise de fonction, la situation est presque catastrophique : la diffusion avait chuté à 68000 exemplaires et ses pertes avaient atteint 1,5 millions de $\mathrm{EE}$. La moyenne d'âge des journalistes était de 51 ans et l'imprimerie de Bûlâq datait de 1928. Un mois plus tard, lors d'une réunion du conseil d'administration, Haykal explique que la priorité est de rééquilibrer les comptes du journal et de planifier les étapes du futur développement. En 1958, il recrute 54 nouveaux diplômés des universités égyptiennes pour les former au métier de journaliste.

Commence alors «la première expérience de formation collective jamais entreprise dans un journal égyptien» (Baybars, s.d, p. 127). À côté d'anciens collègues qui l'ont suivi, Haykal essaye de réunir des noms aussi prestigieux que ceux de Tawfîq al-Hakîm, Naguib Mahfouz, Ahmad Bahâ' al-Dîn ou Yûsuf Idrîs. À la fin de l'année, et pour la première fois depuis dix ans, les gains du journal atteignent $60000 \mathrm{fE}$. Il forme alors une commission chargée d'étudier les possibilités de modernisation du journal. Il envoie des collaborateurs dans différentes grandes maisons de presse dans le monde afin de s'inspirer de leur modèle de développement. Au début de 1960, il consacre 10 $000 \mathrm{EE}$ à l'étude du projet de construction de nouveaux locaux. Le chantier commence le $1^{\text {er juin }} 1963$ et l'inauguration du nouveau bâtiment a lieu le 10 janvier 1969. Il crée également un Centre d'études politiques et stratégiques, un centre de traitement de l'information, un centre de microfilms... Le dynamisme d'al-Ahrâm "l'amène à devenir progressivement le centre d'une entreprise commerciale florissante» (Aulas, 1977, p. 339). Le tirage du journal passe de quelque 100000 exemplaires en 1958 à 423000 en 1974 et le total des gains de l'entreprise atteint 4 millions de $\mathrm{EE}$ en 1968. 
50 Haykal est-il l'idéologue du régime? Il refuse tous les postes officiels qui lui sont proposés ainsi que la direction des journaux porte-parole de la Révolution. Mais il est trop proche de Nâsir pour être un simple observateur. Préférant garder cette importance politique officieuse mais réelle, il ne déroge à cette règle que quelques mois avant la mort de Nâsir en acceptant enfin" le poste de ministre de l'Orientation nationale". Bi-sarâha (En toute franchise), son article hebdomadaire dans al-Ahrâm, augmente le tirage du journal le vendredi qui passe de 700000 à un million d'exemplaires. Il est lu sur les ondes de La voix des Arabes. Cet intérêt du public comme des chancelleries et ambassades n'est pas fortuit: chacun espère y déceler les intentions et réflexions du Zacim. Il n'est plus un secret qu'avant l'écriture de cet article, Haykal a un long entretien téléphonique avec Nâsir.

51 Cet enchevêtrement des rôles entre le journaliste qui observe et enregistre et l'acteur qui joue une part active dans la prise de décision lui vaudra toutes les critiques et jalousies. Il est chargé d'écrire les discours les plus importants. Les événements du 5 juin 1967 (la défaite) illustrent bien ce rôle de bras droit: Nâsir le consulte sur la décision à prendre, lui demande de rédiger son discours de démission et informe ses proches collaborateurs que durant les quelques heures où il s'enferme pour réfléchir, toutes les décisions devront passer par Haykal. Une ligne téléphonique directe relie les deux hommes. Nâsir donne l'ordre de lui envoyer une copie de tous les documents officiels de l'État. Quelques années après la mort du leader, cet auteur prolifique multiplie les publications qui, documents à l'appui, donnent sa version des faits restés obscurs". "Pseudo-historien", "manipulateur de l'histoire", "acteur à la mémoire sélective » tels sont les qualificatifs employés par ses nombreux adversaires.

Après la défaite de 1967, c'est Hasanayn Haykal qui donne le signal du début d'une remise en cause du système et de l'autocritique. Beaucoup de ses écrits lui valent les ressentiments de différents responsables, surtout ceux du service des renseignements. Il est même victime d'une opération d'intimidation (des coups de feu sont tirés dans sa direction alors qu'il se trouve en face du bâtiment du journal). Appuyé par Nâsir, Haykal se défend contre les dirigeants des institutions visées. Mais à la mort du leader, et malgré une bonne entente au départ entre Sâdât [Sadate] et lui, les «centres de pouvoir ", d'après la version de Haykal, seraient parvenus à leur fin, à savoir son éviction, symbole fort du régime nassérien, d'al-Ahrâm.

53 Entre 1952 et 1970, ses écrits suivent les évolutions de la situation interne. Après la Révolution, il consacre ses articles à défendre Nâsir et justifier toutes les mesures qu'il prend. Une fois consolidées les assises du pouvoir du raïs, Haykal se tourne vers des questions liées à la position de l'Égypte dans le monde : ses voisins, Israël et l'Occident. Après la défaite de 1967, c'est sur une critique de tous les aspects de la situation intérieure que portent ses articles. Évidemment ces limites qui sont fictives permettent de dégager les grandes lignes de l'orientation de ses écrits mais, en réalité, les choses ne sont pas toujours aussi tranchées.

54 En presque deux décennies à la tête d'al-Ahrâm, Muhammad Hasanayn Haykal écrit plus de 1500 rubriques Bi-sarâha. Entre août 1957 et juin 1967, on peut classer ses écrits en huit catégories. Ses attaques sont adressées à ce qu'il était convenu d'appeler les régimes arabes "conservateurs" ou "réactionnaires" (les pays du Golfe surtout l'Arabie saoudite et la Jordanie) et également les régimes révolutionnaires arabes opposés à Nâsir (l'Irak de 'Abd al-Karîm Qâsim et l'Algérie de Boumediene); il critique violemment la politique occidentale américaine (tout en prônant une stratégie 
d'évitement) qu'il considère comme responsable de l'orientation pro-soviétique des pays du tiers-monde; il défend avec ardeur la politique de non-alignement, seule chance pour ces pays de se faire une place entre les deux géants et ne pas en être tributaires; Israël fait partie de ses préoccupations premières et il s'efforce d'analyser la nature de la société israélienne, son système politique et militaire ; il suit de près les événements dans les pays arabes soutenus par le régime nassérien à savoir le Liban, la Syrie ainsi que le Yémen révolutionnaire de al-Sallâl; les affaires intérieures sont également traitées dans ses écrits en fonction de l'ordre du jour: le rôle des intellectuels au sein du régime révolutionnaire, les problèmes du secteur public, la nature de la démocratie dans une société socialiste et le rôle de la presse, le pacte national et la nécessité de réorganiser l'Union socialiste ; enfin, Nâsir comme exemple de ses théories sur le rôle de l'individu ou du héros dans l'histoire

Avant la défaite de 1967, Haykal commence une série d'articles sur la dégradation progressive des relations entre l'Égypte et les États-Unis, alliés d'Israël. Il prédit l'imminence d'une guerre avec Israël et la nécessité de s'y préparer. Le 2 juin, il écrit que la guerre est «une question de quelques heures». Son premier article après la défaite (le 9 juin 1967) est intitulé "L’ultime degré de la violence ", qu'il clôt par cette phrase : «Par honnêteté, je ne peux pas dire que nous étions sans fautes... mais par honnêteté je peux dire qu'en 1956, Israël est venu à nous avec la France et la GrandeBretagne deux jours après... et en 1967, Israël est venu à nous avec les États-Unis au moins deux mois avant » (Hammûda, 2000, p. 318).

À compter de cette date, il se lance dans une critique de certains aspects du régime. On lui doit quelques formules restées célèbres telles que les « centres de pouvoir » ou « les visiteurs de l'aube ». Il consacre plus de 25 articles aux questions de la démocratie, de la liberté d'expression, des arrestations arbitraires, de l'Union socialiste devenue un État dans l'État... Il plaide pour un régime plus ouvert, un meilleur respect des lois par les responsables. Dés le moment où il a abordées ces questions, il devient alors possible pour d'autres intellectuels de débattre de ces questions. "Malgré quelques tentatives [...], on peut s'étonner que les journalistes, autrefois si critiques dans l'opposition, ne tentent pas davantage leur chance pour forcer les portes de la liberté d'expression à un moment où cela leur est possible »(Aulas, 1977, p. 341).

Doit-on en déduire que l'autoritarisme nassérien a été intériorisé par les journalistes et plus généralement par les intellectuels? Faut-il y voir le signe que les journalistes, par manque de pratique, ne se font plus confiance en tant qu'acteurs capables d'imposer les termes du débat public et d'en être la source? C'est sur cette revalorisation que vont jouer les régimes post-nassériens qui ont reçu en héritage un État centralisateur fort mais qui ont depuis habilement su accorder plus de prérogatives à la société civile en feignant l'autonomisation du champ du débat public.

À la presse nationale (sihâfa qawmiyya), héritage de la période nassérienne, se sont ajouté une presse d'opposition (sihâfa mucârida) et une presse indépendante (sihâfa mustaqilla). Quel espace du champ public occupe chacune d'elles? Quelle signification revêt le fait que les acteurs (journalistes et plus largement intellectuels) passent de l'une à l'autre selon ce qu'ils veulent dire ? Il en découle que les « limites du dicible " $\mathrm{y}$ sont mouvantes.

En même temps, si les journalistes continuent de jouer le rôle d'intermédiaire entre le pouvoir et la société, il n'en demeure pas moins que les règles du jeu se sont sensiblement transformées. La société les interpelle également et ils sont en charge de 
transmettre au pouvoir ses requêtes et d'exposer ses maux afin de l'aider à trouver des solutions.

Cet article doit beaucoup aux conseils judicieux de Sarah Ben Nefissa. Qu'elle en soit chaleureusement remerciée. Le travail de Layla ‘Abd al-Magîd m'a été d'une grande utilitée ('Abd al-Magîd, s.d.).

\section{BIBLIOGRAPHIE}

‘Abdu Ibrâhîm, 1982, Tatawwur al-sihâfa al-misriyya 1798-1981 [Évolution de la presse égyptienne 1798-1981], Le Caire, Mu'assasat sigill al-carab, 4 édition, 376 p.

cAbd al-Magîd Laïla, s.d., Tatawwur al-sihâfa al-misriyya min 1952 ilâ 1981 [Évolution de la presse égyptienne de 1952 à 1981], Le Caire, Al-carabî li-l-nashr wa-l-tawzî́c, 125 p.

‘Abdel-Malik Anouar, 1962, Égypte, société militaire, Paris, Éditions du Seuil, 380 p.

Aulas Marie-Christine, 1977, « Regards sur la presse égyptienne », in Aulas M.-C. (dir.), L'Égypte d'aujourd'hui, permanence et changements 1805-1976, Paris, Éditions du CNRS, p. 331-343.

Balle Francis (dir.), 1998, Dictionnaire des médias, Paris, Larouse, « Les référents ».

Fargues Philippe, 1994, « Note sur la diffusion de l'instruction scolaire d'après les recensements égyptiens », in Égypte-Monde arabe n 18-19, L'Éducation en Égypte, Le Caire, CEDEJ, p. 115-167.

Gamil Sayyâr al-, 2000, Tafkîk Haykal : mukâshafât naqdiyya fî ishkâliyyât Muhammad Hasanayn Haykal [La déconstruction de Haykal : révélations critiques sur les problématiques de Muhammad Hasanayn Haykal], Aman, al-Ahliyya li-l-nashr wa-l-tawzî́c, 624 p.

Hammûda cÂdil, 2000, Haykal al-hayât, al-harb, al-hubb, huwa wa cAbd al-Nâsir [Haykal, la vie, la guerre, l'amour, avec Nâsir], Le Caire, Al-Fursân li-l-Nashr, 398 p.

Hamzah 'Abd al-Latîf et Sharaf 'Abd al-cAzîz, 1998, Adabu-l-maqâla al-suhafiyya fî misr, D. Muhammad Husayn Haykal fî garîdat al-Siyâsâ al-yawmiyya [L'essai journalistique en Égypte, D. Muhammad Husayn Haykal et le quotidien al-Siyâsâ], Vol. IX, Le Caire, GEBO, 308 p.

Haykal Muhammad Hasanayn, 1984, Bayna al-sihâfa wa-l-siyâsa [Entre presse et politique], Beyrouth, Sharikat al-matbûcât li-l-tawzî̀ wa-l-nashr, $452 \mathrm{p}$.

Haykal Muhammad Husayn, 1990, Muzakkirât fî al-siyâsa al-misriyya [Souvenirs de politique égyptienne], 3 Vol., Dâr al-macârif, 931 p.

Haykal Muhammad Husayn, 1996, Muzakkirât al-shabâb [Souvenirs de jeunesse], Le Caire,GEBO, Almaglis al-Aclâ li-l-thaqâfa, 375 p.

Jacquemond Richard, 2001, "Les limites mouvantes du dicible dans la fiction égyptienne » in La censure ou comment la contourner. Dire et ne pas dire dans l'Égypte contemporaine, EMA, n 3, CEDEJ, $\mathrm{p}$. 63-83.

Khalil Nagwa Husayn, 1995, Al-mugtamac al-misrî qabla al-thawra fî-l-sihâfa al-misriyya 1945-1952 [La société égyptienne avant la révolution à travers la presse égyptienne 1945-1952], Le Caire, GEBO, $362 \mathrm{p}$. 
Laurens Henry, 1995, « Élite et réforme dans l'Égypte du XIX siècle », in Roussillon Alain (Dir.), Entre réforme sociale et mouvement national, identité et modernisation en Égypte (1882-1962), Le Caire, CEDEJ, p. 91-102.

Meijer Roel, 2000, al-Bahth cani-l-hadâtha, al-fikr al-siyâsî al-cilmânî al-lîbarâlî wa-l-yasârî fî misr 1945-1958 [La quête de la modernité, la pensée politique, laïque, libérale et de gauche en Égypte 1945-1958], Traduction de Sharîf Yûnis, Le Caire, Mîrît li-l-nashr wa-l-maclûmât, 320 p, Collection « Mukhtârât Mîrît ».

Muhammad Sayyid Muhammad, 1996, Haykal wa-l-Siyâsa al-usbûciyya [Haykal et al-Siyâsa alusbûciyya], Le Caire, GEBO, 390 p.

Neveu Éric, 2001, Sociologie du journalisme, Paris, La Découverte, « Repères », 123 p.

Sabrî Mûsâ, 1992, 50 câman fí qitâr al-sihâfa [50 ans dans le train de la presse], Dâr al-shurûq, 1018 p.

Saydawi Riyâd Al-, 2000, Haykal aw al-milaff al-sirrî li-l-zâkira al-carabiyya [Haykal ou le dossier secret de la mémoire arabe], Le Caire, $2^{2}$ édition, Madbûlî, 290 p.

Shalabi Gamâl Al-, 1999, Muhammad Hasanayn Haykal : istimrâriyya am tahawwul ? [Muhammad Hasanayn Haykal : continuité ou rupture ?], Traduction de Hayât al-Huwayyik cAtiyya, Beyrouth, Al-mu'assassa al-carabiyya li-l-dirâsât wa-l-nashr, 366 p.

\section{NOTES}

1.Malgré la similitude des noms, il n'existe aucun lien de parenté entre ces deux personnages.

2.Cf. la première partie de ce travail.

3.Sauf pendant une période de 6 mois durant laquelle il a été ministre de l'Orientation nationale. $C f$. la deuxième partie de l'article.

4.Il écrit régulièrement un article dans une publication mensuelle, Wighât nazar. 5.Son expérience lui inspire l'écriture de ses mémoires. Il y décrit non seulement la France, mais tous les pays européens qu'il visite en plus de quelques articles datant de la même période, dont une réflexion sur les réformes à apporter au Code du statut personnel et au système fiscal égyptiens.

6.(1872-1963). En 1906, il crée le parti d'al-Umma, le plus important porte-parole de la bourgeoisie libérale et laïque. Considéré comme le " professeur » d'une génération de libéraux égyptiens à l'influence assez importante jusque dans les années 1930, il réussit à élaborer une version égyptienne du libéralisme, adaptée à sa classe sociale et à ses intérêts cosmopolites. Il définit la umma selon des considérations « civiles » qui comprennent donc la suprématie de l'appartenance à un territoire sur fond d'égalité de tous devant la loi. Mais il entend par umma uniquement les membres des grandes familles et la responsabilité du pouvoir doit, selon lui, revenir à ceux qui ont des intérêts à défendre. Pour plus de détails sur la pensée, voir Meijer R., 2000, p. 38 ; Ibrâhîm cAbdû, 1982, p. 180-181; et, sur la vie du personnage, Hamza cAbd al-Latîf, 1995, Adabu-l-maqâla al-suhfiyya fî misr [L'essai journalistique en Égypte], T II, GEBO, p. 571-780. 7.Cette revue littéraire, parue en mai 1915, a rassemblé les anciens d'al-Siyâsa dont Mustapha 'Abd al-Râziq, Taha Husayn, Mansûr Fahmî et Abd al-Hamîd Hamdî. C'était une continuation de leur ancienne revue mais avec quelques améliorations. Y ont contribué : Mahmûd Taymûr, Ahmad al-Sâwî Muhammad, Mustapha Râchid Rustum, 
‘Azîz Abâza, Mahmûd cAzmî, Nîllî ‘Azab... Elle s'est arrêtée en 1921 à cause des différents politiques qui ont opposé ses rédacteurs.

8.Al-Ahrâm, al-Muqtataf et al-Bayân publient des articles portant sur la littérature, la politique et la société.

9.Zaynab, Le Caire, Dâr al-Macârif, 5e édition, 1992, p. 8.

10.Hayât Muhammad, 1935 ; Fî manzil al-wahy, 1937 ; Al-Siddîq Abû Bakr, 1942 ; Al-Fârûq cUmar, 1945.

11.Il a été très influencé par la théorie de Thomas Carlyle sur Les Héros et le culte des héros, selon laquelle les grands hommes jouent un rôle moteur dans l'histoire de l'humanité, Mémoires politiques, Vol. I, p. 31.

12.Pendant ses différents passages au ministère de l'Instruction publique, il essaye d'obtenir des fonds pour la création d'écoles primaires supplémentaires car il remarque qu'en 1938, à peine un quart des enfants sont scolarisés. Pour plus de détails, voir le chapitre 3 de ses Mémoires politiques, Vol II, p. 78-113.

13.En voyant l'agitation de la rue lors des manifestations de 1919, il dit « l'animal parlant a été libéré de tous ses liens » (1990, vol. I, p. 76). Face à la polémique que provoque la parution de La libération de la femme de Qâsim Amîn, Haykal s'étonne de cette réaction hostile et dit : « j'ai commencé à comprendre que [se laisser entraîner par] les foules est une voie facile mais qui mène la plupart du temps à l'erreur » (1990, vol. I, p. 27).

14.Créé début 1919 avec Mustafâ cAbd al-Râziq, Mansûr Fahmî, Mahmûd ‘Azmî, cAzîz Mîrham.

15.Par Muhammad Mahmûd, cAdlî Yaghin, Muhammad cAli cAllûba, Ahmad Lutfî alSayyid et d'autres le 30 octobre 1922. Ils n'étaient pas d'accord avec Sacd Zaghlûl sur la façon de parvenir à l'indépendance de l'Égypte.

16.Non seulement ils s'allient avec le parti d'al-Ittihâd (soutenu par le roi) pour former un gouvernement (celui de Ahmad Zîwar 13 mars 1925 - 7 juin 1926), mais ils ne protestent pas contre la dissolution de l'Assemblée à majorité wafdiste une deuxième fois pendant la même législature. En juillet 1928, les libéraux sont à l'origine de la dissolution de la Chambre pour une période de trois ans.

17.Créée en 1926. Le premier numéro paraît le 26 mars et le dernier le 28 mai 1949. Elle a été suspendue de 1931 à 1937.

18. Beaucoup de ceux qui ont participé à al-Sufûr en plus de May, Fikrî Abâza, al-Mâznî, cAli Mahmûd Tih, Ibrâhîm Nâgî, cAbd al-cAzîz al-Bishrî, cAzîz Mîrham... Pour plus de détails, voir Muhammad, 1996, p. 277-281.

19.Ministre de l'Hygiène publique dans le premier gouvernement de Muhammad Mahmûd 30 décembre 1937-5 avril 1938 ; cinq fois ministre de l'Instruction publique : dans les deuxième et troisième gouvernements de Muhammad Mahmûd 27 avril 193812 août 1939 ; dans les premier et deuxième gouvernements de Husayn Sirrî 15 novembre 1940-2 février 1942 ; dans le premier gouvernement de Ahmad Mâhir 9 octobre 1944-15 janvier 1945.

20.Issu d'une scission du Wafd, le Parti saadiste est fondé en 1930 par Hâmid al-Bâsil ; Ahmad Mâhir et Mahmûd Fahmî al-Nuqrâshî créent le Groupe saadiste en 1937. Les partis du roi : parti al-Ittihâd (1925), dont le président est Yihyâ Ibrâhîm et plus tard Hilmy Issa, puis al-Shacb (1930) d'Ismâîl Sidqî (ancien wafdiste devenu un de ses plus grands adversaires) ; les hommes politiques indépendants qui représentent les milieux d'affaires et la banque, la grande industrie, les cadres dirigeants de la technocratie. 
21.Ministère de Sacd Zaghlûl 28 janvier 1924-24 novembre 1924 ; 8 ministères de Mustafâ al-Nahhâs, répartis entre la fin des années vingt et le début des années cinquante.

22.Avocat diplômé de la Faculté de droit en 1903, il écrit pour al-Garîda pendant plusieurs années. Il traduit notamment des œuvres littéraires françaises. Puis il assume la rédaction d'al-Ahâlî, créé à Alexandrie en 1910 par des notables. Publié pour la première fois au Caire en 1921, il est un ardent défenseur de la politique de Sacd Zaghlûl. Après de multiples péripéties, il parvient à publier al-Balâgh, plusieurs fois suspendu. Une édition hebdomadaire paraît à partir du 26 novembre 1926 en 40 pages et traite de sujets différents, allant de la politique à la littérature, de l'économie à la société et notamment à la question féminine.

23.Ancien rédacteur du journal al-Mu'ayyid du shaykh cAli Yûsuf qui, avant la Première Guerre mondiale, prônait un rapprochement avec l'empire ottoman, seule façon de se débarrasser des Anglais. Ahmad Hâfiz ‘Awad écrit dans différents journaux avant de créer Kawkab al-Sharq.

24.D'après Wâdî Taha Imrân, Al-ductûr Muhammad Husayn Haykal, hayâtuhu wa turâthuhu al-adabî [Le docteur Muhammad Husayn Haykal, sa vie, son œuvre], Magistère, Université du Caire, Faculté de Lettres, département de langue arabe, section de littérature arabe, p. 104.

25.En avril 1925, ce cadi au tribunal religieux de Mansoura entreprend d'établir dans son livre L'Islam et les fondements du pouvoir que la thèse de la séparation de l'Église et de l'État est conforme aux enseignements du Coran et de la Sunna. C'était une tentative audacieuse pour prouver la légitimité de la suppression du califat par Mustaphâ Kâmil. Le 12 août, le Grand Conseil de l'Université d'al-Azhar condamne cette thèse comme contraire à l'orthodoxie. Le shaykh est exclu du corps des culama et déclaré déchu de sa charge de cadi.

26.En 1926, dans son livre Al-shi'r al-gâhilî [La poésie anté-islamique], il se propose d'appliquer à l'étude de la littérature la méthode critique de Descartes. Il n'hésite pas à suspecter l'authenticité de la poésie pré-islamique et à mettre en doute quelques-uns des postulats religieux admis par l'orthodoxie.

27.Mahmoud 'Azmî appelle à l'union entre l'Égypte et la Palestine tandis que Haykal plaide pour le renforcement des relations économiques entre les pays du Moyen-Orient. 28.Taha Husayn lui dit : « Je n'ai écrit cet article que pour que tu y répondes en adoptant la position contraire qui postule que les guerres n'ont cessé de détruire et que c'est la bêtise qui pousse l'humanité vers la guerre ", (Haykal, 1990, Vol. I, p. 66).

29.Thèse réfutée par Haykal qui dit : «Il est vrai que nous n'étions pas riches mais nous n'étions pas pauvres non plus ", Shalabî Gamal al-, 1999, p. 31, d'après Nâsir Munîr, 1979, Press Politics and Power: Egypt's Heikal and al-Ahram, Iowa, The State University press ; Cf. Sabrî Mûsâ, 1992, p. 790.

30.Malgré les nombreuses références à ces études dans les ouvrages qui contiennent la biographie de Hasanayn Haykal, toutes restent floues et ne mentionnent pas le nom de l'école française ni l'institution qui lui aurait délivré son diplôme par correspondance. 31.Information démentie par ses adversaires, notamment Sabrî Mûsâ, 1992, p. 790-791. 32.Des forces militaires encerclent le palais Abdîn et le roi est obligé de céder aux exigences britanniques concernant la formation du gouvernement par Mustafâ alNahhâs.

33.Pour plus de détails, voir Abdel-Malek A., 1962, p. 34. 
34.Le 9 février 1946, une manifestation étudiante est contrée par la police ; al-Nuqrâshî est contraint de démissionner. Le 21 février 1946, de nouvelles manifestations au Caire et à Alexandrie font des victimes sous le gouvernement de Ismâîl Sidqî.

35.Il y a même eu un projet visant à limiter la propriété à 50 feddan, proposition de Ibrâhîm Shukrî du parti Misr al-fatât (Khalîl, 1995, p. 354).

36.Sur encouragement de Scott Watson, journaliste américain, longtemps correspondant de guerre. Haykal suit son exemple et part à El-Alamein y décrire la guerre qui s'y déroule d'un point de vue égyptien. Il reste au journal jusqu'en 1944. 37.Il y travaille de 1944 à 1946.

38.(1896-1976). Licencié en droit, il est fonctionnaire pendant quelques années tout en étant en contact avec le monde de la presse et du théâtre. Lors de la création de Rûz alYûsuf, il démissionne en 1928 pour se consacrer à la rédaction de la revue. Il est un acteur important du passage de la revue du champ littéraire et artistique au champ politique. En 1934, il crée sa propre publication, Akhir Sâca, qui est le point de départ d'une nouvelle école journalistique en matière de revue hebdomadaire. En 1936, il contribue à la création du journal al-Misrî mais en cède ses parts en 1938. Faisant face à des difficultés financières, il vend sa revue en 1946 à ses anciens élèves, les frères Amîn. 39.Les jumeaux cAlî (1914-1976) et Mustafâ (1914-1997) Amîn. Après avoir travaillé l'un et l'autre pour plusieurs journaux dont Rûz al-Yûsuf, Akhir Sâca, al-Ithnayn, ils créent en 1944 l'une des premières maisons de presse égyptiennes indépendantes, Akhbâr alYawm. Publication au succès immédiat, leur maison est prolifique : ils rachètent Akhir Sấa en 1946, créent la revue Akhir Lahza en 1948, la revue al-Gîl al-Gadîd en 1951, puis le journal Al-Akhbâr en 1952. Suspectés dès le lendemain de la Révolution de 1952, la suite de leur carrière n'est qu'une succession de conflits-réconciliations avec le pouvoir politique. Mustafâ est emprisonné pendant 9 ans alors que son jumeau est en exil forcé. 40.Il est reporter de guerre pendant cinq ans, de 1946 à 1951. Il couvre les conflits les plus importants : la guerre en Grèce et dans les Balkans, les coups d'État militaires en Syrie, l'assassinat du roi Abd Allah à Jérusalem, les mesures de Mossadegh en Iran en 1951, la guerre de Corée mais beaucoup soutiennent que les reportages écrits pendant la première guerre israélo-arabe de 1948 l'ont fait connaître et par le grand public et par les hommes politiques.

41.Avant Nâsir, il est en contact avec le monde de la politique. al-Hilâlî lui demande conseil à propos de la formation de son gouvernement et al-Nuqrâshî le convoque pour s'informer de la situation en Palestine (Shalabî 1999, p. 33). Sayyâr al-Gamîl remet en cause cette histoire (p. 65).

42.Articles qu'il écrit avant que l'Égypte ne prenne part au conflit.

43. Quelques années plus tard, la loi 156 de 1960 vient réorganiser (ou nationaliser, selon ses détracteurs) la presse.

44. Qui titre un de ses éditoriaux « Non à la démocratie ».

45.Pendant la signature de l'accord Sidqî-Beven en 1946, Akhbâr al-Yawm titre son éditorial « Signons-le en la maudissant » et Akhir Sấa, rachetée par la maison des frères Amîn et dont Haykal assume la rédaction, intitule le sien « Si c'est pour le maudire, pourquoi le signer?».

46.Cette version est citée dans les ouvrages de Hasanayn Haykal (1989), Gamal alShalabî (1999) et Saydâwî (2000). Par contre, Mûsâ Sabrî dit qu'il s'est accordé beaucoup trop d'importance dans ces événements. En plus, Haykal aurait donné quatre versions différentes de sa première rencontre avec Nâsir (p. 806-807). 
47.Fondé par les frères Taqla, des syro-libanais émigrés en Égypte à la fin du XIX siècle, le premier numéro de cet hebdomadaire paraît le 5 août 1976 et se transforme en 1881 en quotidien. Pendant longtemps précurseur en matière d'équipement (il est le premier à introduire la linotypie et la rotative en Égypte), il se caractérise par sa politique de modération et de neutralité dans le but de ne provoquer aucun gouvernement quelle que soit sa tendance.

48.Il avait refusé le poste de ministre de l'Orientation nationale quatre fois : à une première reprise en 1956, deux fois entre 1958 et 1961, puis encore une fois après la défaite de 1967.

49.26 avril-18 octobre 1970.

50.(1984), Bayna al-sihâfa wa-l-siyâsa [Entre presse et politique], Beyrouth, Sharikat almatbûcât li-l-tawzî̀ wa-l-nashr, 452 p ; (1985), Qissat al-suwîs [Le récit de Suez], Sharikat al-matbûcât li-l-tawzî‘'wa-l-nashr ; (1986) Li-misr lâ li-‘Abd al-Nâsir [Pour l'Égypte, non pour Nâsir], Sharikat al-matbûcât li-l-tawzî́wa-l-nashr ; (1986), Milaffât al-suwîs : harb althalâthîna sana [Les dossiers de Suez : la guerre de trente ans], Vol. I, Markaz al-Ahrâm li-l-targama wa-l-nashr ; (1988), Sanawât al-ghalayân : harb al-thalâthîna sana [Les années d'effervescence : la guerre de trente ans], Vol II, Markaz al-Ahrâm li-l-targama wa-lnashr ; (1990), Al-'infigâr 1967 : harb al-thalâthîna sana [L'explosion 1967 : la guerre de trente ans], Vol. III, Markaz al-Ahrâm li-l-targama wa-l-nashr.

51.Expression empruntée à R. Jacquemond, 2001.

\section{INDEX}

Mots-clés : histoire, journalisme, Haykal (Muhammad Husayn), Haykal (Muhammad Hasanayn), âge libéral, Nasser (Gamal Abdel)

\section{AUTEUR}

SONIA TEMIMI

Cedej 